\title{
Research of Communication Technology on IOT for High-Voltage Transmission Line
}

\author{
Shao-Lei Zhai ${ }^{\mathrm{a}}$, Dong-Sheng Zhao ${ }^{\mathrm{b}}$, Zhen Wang ${ }^{\mathrm{c}}$, Yi Zhang ${ }^{\mathrm{c}}$ \\ ${ }^{a}$ Yunnan Provincial Electric Power Test \& Research Institute (Group) Co., Ltd, Kunming City, China, 650217 \\ ${ }^{b}$ School of Electric Power, South China University of Technology, Guangzhou City, China, 510641 \\ ${ }^{c}$ Kunming University of Science and Technology, Kunming City, China, 650217
}

\begin{abstract}
This paper presents a three layered network construction of Internet of Things (IOT) communication method for high-voltage transmission line which involves the wireless self-organized sensor network (WSN), optical fiber composite overhead ground wire (OPGW), general packet radio service (GPRS) and the Beidou (COMPASS) navigation satellite system (CNSS). The function of each layer of network, application deployment and management of energy consumption are studied. The method can meet the needs of interconnection between the monitoring center and terminals, reduce the terminals' GPRS and CNSS configuration and OPGW optical access points, and ensure the on-line monitoring data transmission real-time and reliable under the situation of remote region, extreme weather and other environmental conditions.
\end{abstract}

Keywords: Internet of things (IOT), high-voltage transmission line, on-line monitoring, CNSS, WSN

\section{Introduction}

The idea of smart grid is promoted with the increasing demands of the distributed energy's access, monitoring ability on the power grid, and the information exchange between the power grid and the users, it is an completely automatic power grid in which all the users and equipments are monitored, and the information exchange at every point from the power plants to the users should be guaranteed. The transmission line monitor system on line is an important part of smart grid and efficient method to improve the management of the production and operation of the power transmission line.

The realization of smart grid depends on the monitoring technologies that are adopted in the power grid and the real-time operation parameters, the internet of things (IOT) which is thought as the nerve ending of the intelligent sensing will become the important means that promotes the development of smart grid. The definition of IOT is the fusion of the information space and physical space, which make all of the things digitization and networked, realize the efficient way to exchange information between articles, articles and person, person and the real word, make all kinds of information technology into social behavior through new service model, it is a higher realm of comprehensive application of the information in human society [1]. It is the development tendency of smart grid that applies the technology of IOT in power system to optimize the operation and stability [2].

High voltage transmission line is the key equipment in power transmission, but the safety of power transfer is threatened by some indefinitely factors such as natural calamities (hoar frost, hurricane, etc.) and deliberate damage (stealing, destroy, etc.) [3]. For these reasons, the on-line monitoring device for the high voltage transmission line is very important to guarantee the operation of power grid. How to send the monitoring data gathered by sensors that are fixed on the transmission line or tower safely and timely to

\footnotetext{
* Manuscript received July 23, 2012; revised August 25, 2012.

Corresponding author. Tel.: +86-871-6345198; fax: +86-871-6345198; E-mail address: zslsd@163.com.
} 
the monitoring center is a very important problem that needs to be solved. Generally the place where the transmission line is set up is far away from cities and sparsely populated, the topographies are varied and complicated, the communication method which can be adopted is limited, at present the choices include general packet radio service (GPRS), optical fiber composite overhead ground wire (OPGW), 3G, etc. [4], these communication technologies can not cover all the areas the transmission line passed, in some area, the radio signal is unstable or even does not work at all, this result in not properly sending the monitoring data back and instability of on-line monitoring data transfer.

The sensory ability and communication stability of on-line monitoring devices can be improved obviously by adopting the technology of IOT. In this paper, the communication technologies in the high voltage transmission line IOT are researched and a layered scheme is proposed.

\section{Communication Architecture of High Voltage Transmission Line IOT}

The communication architecture of IOT for high voltage transmission line is shown in Fig.1, it is divided into three layers, the first layer is called sensor layer, which is composed by sensors, on-line monitoring terminals and wireless routers, the sensors are responsible for sensing the physical information, the on-line monitoring terminals are responsible for gathering the monitoring data from the sensors, and the wireless routers are responsible for building the multi hop wireless network, through which the monitoring terminals can exchange data; the second layer is called fiber communication layer, the fibers in the OPGW cable are used as the communication path, the data gathered by monitoring terminals is send to the sink terminal connected to the OPGW by wireless router, and transfer to the data center; the third layer is the composed by GPRS network and the Beidou (COMPASS) navigation satellite system (CNSS), it is used in those place where there is no OPGW or the OPGW does not work well, in this layer the data gathered by monitoring terminals is send to the sink terminal equipped with GPRS module and CNSS module, the GPRS module is the priority choice for data transfer and the CNSS module only work when the GPRS module can't work as normal.

\subsection{Sensor layer}

The full perception on the physical world is the basic character of IOT. Because the transmission line extends as far as hundreds, even thousands of kilometers, the topographies are varied and complicated, so the objects need monitoring are of great number, such as the pull and temperature of the transmission line, the temperature and humidity of the microclimate around the transmission line, etc.. Generally the type of the sensors is chose according to the topography where the transmission line is set, the sensors and monitoring terminals which are fixed on the towers or transmission lines construct the basic sensing area to get the full sense of the environment information.

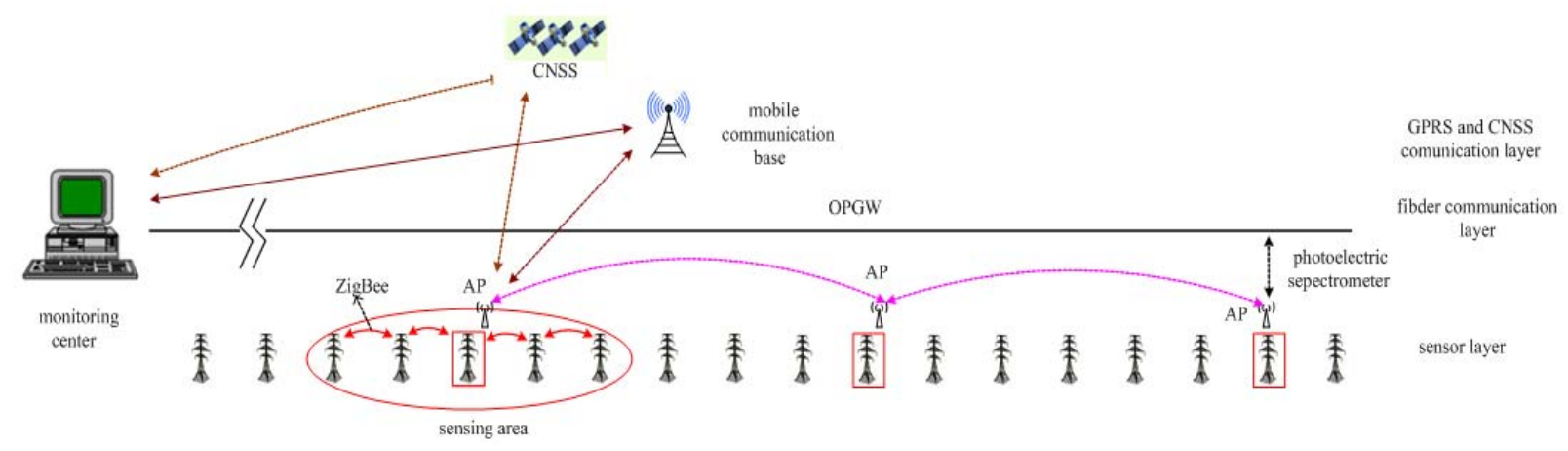

Fig.1. Architecture diagram of the IOT for high-voltage transmission line.

In the construction of basic sensing area, the terminals in neighbor towers communicate with each other using ZigBee technology, in every basic sensing area, a central terminal which equipped with wireless router communicate with other basic sensing areas, and these central terminals construct the chain wireless network. In order to reduce the number of GPRS modules and CNSS modules, all the data 
of every monitoring terminal is transferred by its neighbor terminals to the sink terminal, which is equipped with GPRS module and CNSS module, or OPGW interface module and is responsible for transferring data to the monitoring center. The number of sink terminals can be set on the situation of the monitoring areas, as Fig. 2 shows. According to the reasonable configuration of the monitoring terminals and sink terminals, the monitoring terminal can transfer data by the chain wireless network when the radio signal is unstable, through this method, the monitoring data can be transferred timely and stably.

The ZigBee technology whose base is the IEEE802.15.4 criterion and the working frequency is 2.4 $\mathrm{GHz}$ or $868 / 928 \mathrm{MHz}$, has been widely used in industrial control, industrial wireless location and other fields for its characters of low speed, low cost, supporting large number of network points, multiple network topology, etc. [5]. In the chain wireless network, the data will be banked-up at some point for the data quantity increasing with the transfer points [6], by the use of wireless routing and high power-gain parabolic antenna, the transmission distance can reach 3 kilometers, the relay times of data is reduced and the data blocking is avoided.

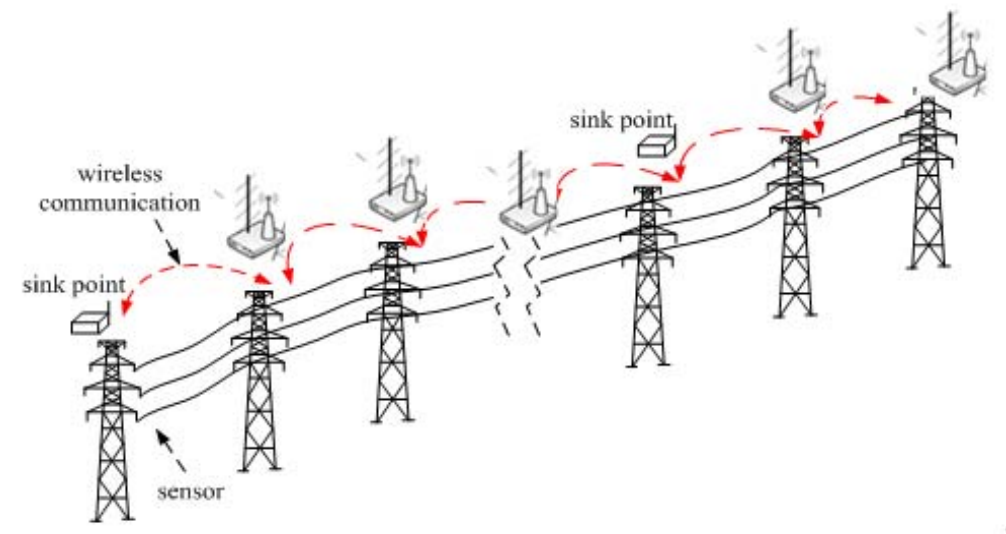

Fig. 2. Architecture diagram of data transfer in line network.

\subsection{Fiber communication layer}

The OPGW is a combination of fiber and overhead ground line, it is an important development direction of special optical cables, and is widely used with the development of power system's optical fiber communication network. The OPGW is set on the top of the towers like other overhead ground line, can be worked as the communication line and the ground line. As the medium of transmitting light signal, the OPGW cable can be used to transmit audio data, video, data, control signal, etc., and build the multichannel broadband communication network. Currently the OPGW fiber cable is fixed in most $500 \mathrm{KV}$ transmission lines, and there are at least twenty four cores in it, so in spite of the use of relay protection, automation, communication, etc., it is adequate for the monitoring terminals' using. The data speed can reach $50 \mathrm{Mb} / \mathrm{s}$.

Generally there is a connector box every two kilometers on the OPGW cable, cooperating with the technology of wireless and multi-hop transfer, the number of connector point can be reduced, the influence for the existing fiber communication is farthest reduced.

\subsection{GPRS and CNSS communication layer}

In some places, the OPGW does not allowed to access, the breakage of the OPGW cores is often occurred in extreme low temperature, and the fiber communication can't be used, under these situation, the GPRS module and CNSS module fixed on the monitoring terminals will start working.

GPRS is the abbreviation of general packet radio service and packet is the way to transmit, it is available for GSM mobile phone users, the transmission speed is between $56 \mathrm{kbps}$ to $114 \mathrm{kbps}$. The CNSS is a navigation satellite system built by China, it is composed by three satellites, the control center and the users, the architecture of communication network of CNSS as Fig. 3 shows. The CNSS service the user all-weather and round-the-clock, quick location, real-time navigation, short message, precise time service 
are its the main function. The CNSS is different from GPS system and GLONASS system for its source positioning system, the user need asking for positioning service from the control center when obtaining the satellite signal, so the user need not calculating the position, but send data to the signal is need. Compared with GPRS system, the CNSS system is available for the users to communicate through short message in spite of location position, this make the communication between users, user and the control center. Our scheme is based on the function of short message to realize the IOT of power transmission line, and the transmission speed is 19200 bps and the message length is 100 byte.

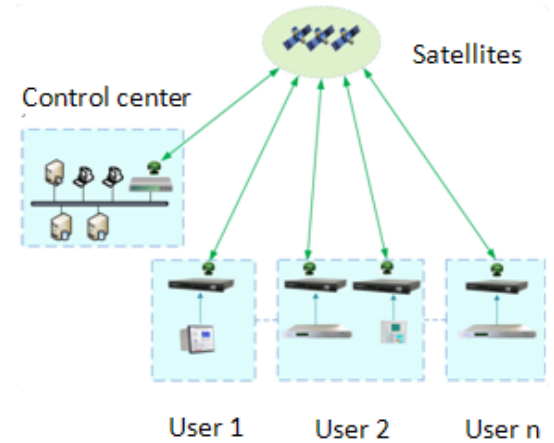

Fig. 3. Architecture diagram of communication using Beidou navigation system.

Through the arrangement of CNSS modules and GPRS modules, the image and video can be transferred by GPRS modules, if the GPRS signal does not cover the sensing area or the signal is not stable, the very important data can be sent by shot message of CNSS.

\subsection{Arrangement of communication devices}

According to the communication scheme of high voltage transmission line this paper mentioned, the monitoring system on transmission line can communicate stably. The arrangement is shown in table 1 , the monitoring data is divided into two types, one type is the data that contain large quantities of information, like the video and high-quality image, the other type is the information like swing frequency, icingthickness, etc. that the quantity of information is about dozens of bytes. When the OPGW access and GPRS are allowed, the first type of information can be transferred, otherwise the second type of information should be guaranteed transfer through CNSS.

Table 1 . The arrangement of communication devices

\begin{tabular}{lll}
\hline Condition & Configuration & Type of data transfer \\
\hline No GPRS signal & CNSS module & Short message \\
\hline GPRS signal is not stable. & $\begin{array}{l}\text { GPRS module, } \\
\text { CNSS module }\end{array}$ & $\begin{array}{l}\text { When GPRS signal is stable, the first type and second type } \\
\text { of data can be transferred, otherwise, short message is used } \\
\text { to transfer. }\end{array}$ \\
$\begin{array}{ll}\text { There are several terminals, and } \\
\text { access to the OPGW is allowed. }\end{array}$ & WSN,CNSS,OPGW, & $\begin{array}{l}\text { Data gathered by WSN and transfer through OPGW,CNSS } \\
\text { is the backup }\end{array}$ \\
$\begin{array}{l}\text { There are several terminals, and } \\
\text { access to the OPGW is not } \\
\text { allowed. }\end{array}$ & WSN,GPRS,CNSS & GPRS is the priority selection, CNSS is the backup \\
\hline
\end{tabular}

\section{Power Management}

Because the special working condition, the on-line monitoring system often powered by battery, which can be charged by solar energy, electromagnetic energy, wind power, etc., but for the limitation of environment and power gathering technology, the monitoring terminal's working time is not long. Improving the battery capacity to extend the terminal working time is an efficient method, but the related research shows that the battery capacity cannot be improved greatly in near future. In the past three decades, the capacity of the battery unit volume only increase less than four times, while the gap between the capacity of the battery and the microprocessor's energy consumption is increasing $20 \% \sim 30 \%$ every 
year [7]. If there is no certain method is adopted to reduce the sensor's energy consumption, the battery will become an important bottleneck of IOT in transmission line, so how to efficient using the battery's energy is an important question.

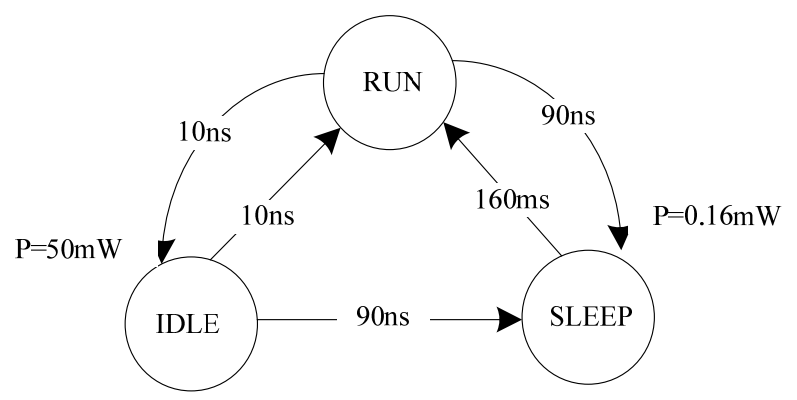

Fig. 4. Switch of power statuses.

Because the sensor module's energy consumption is very low, so how to reduce the microprocessor and the communication module's energy consumption should be considered. The sensor's work status can be divided as multi statuses (active, idle, sleep), that has different energy consumption, and the different status can be switched with certain energy cost [8], as Fig. 4 shows. The monitoring system work periodically, this can be researched to find optimized scheduling algorithm, thus the battery's energy can be used reasonably to lengthen the sensor's working time.

Furthermore, the working feature of sensor network can also be used to save energy. For example, sporadic event make the sensor node work in idle listening state, which is a waste of battery energy. If appropriate schedule scheme is adopted to make the sensor sleep, the energy is saved.

The test shows, if the terminals work under proper sleeping mechanism, the daily average energy consumption is less than 7 watts, which means the terminals can work independently with the proper energy harvesting unit. The terminals energy consumption is shown in table.2.

Table 2. The terminals energy consumption

\begin{tabular}{lcc}
\hline Device name & Standby power $(\mathrm{W})$ & Sending power $(\mathrm{W})$ \\
\hline CNSS module & 3.5 & 24 \\
\hline Wireless module & 0.3 & 1.1 \\
\hline Terminal and sensor & 0.96 & 7.5 \\
\hline
\end{tabular}

\section{Conclusion}

In this paper, the three layered scheme of high voltage transmission line IOT is proposed, the character and working principle of different layer are researched, and the sensor's power management is also studied. We conclude that the monitoring terminals can communicate with each other, and the requirement for time and stable of data transfer is guaranteed in remote area or extreme weather by the combination of GPRS, CNSS and OPGW.

\section{Acknowledgements}

This work has been supported by the state 863 project under grant No. 2011AA05A120.

\section{References}

[1] Sun Q, Liu J, et al. Internet of things: summarize on concepts, architecture and key technology problem. Journal of Beijing University of Posts and Telecommunications, 2010; 33(3):1-9.

[2] Li X,Gong Q, Qiao H. The application of IOT in power system. Power System Protection and Control, 2010; 38(22):232-236.

[3] Li C, Lu Y, Cui X, et al. Research issues for safe operation of power grid in china under ice-snow disaster. Power System 
Technology, 2008; 32(4):14-22.

[4] The Communication Protocol of China Southern Power Grid Transmission Line Integrated Operation Condition of On-Line Monitoring System. Q/CSGXX-2011.

[5] Chen S. The study of key communication technologies for wireless sensor networks based electricity tower monitoring system. Dalian Maritime University, 2011.

[6] Sun LM, Li B, Zhou X. A survey of congestion control technology for wireless sensor networks. Journal of Computer Research and Development, 2008; 45(1):63-72.

[7] Wang X-D. Energy efficient algorithm research in wireless sensor networks. MS thesis. Zhejiang University, 2007.

[8] Yu Y. Research on low power model for embedded system. MS thesis. Zhejiang University, 2007. 